\title{
Research on Intelligent Dispatching System Management Platform for Construction Projects Based on Digital Twin and BIM Technology
}

\author{
Heng Sun ${ }^{1}{ }^{1}$ and Zhe Liu' \\ ${ }^{1}$ School of Digital Construction, Jilin University of Architecture Technology, Changchun 130000, Jilin, China \\ ${ }^{2}$ School of Economics and Management, Jilin Jianzhu University, Changchun 130000, Jilin, China \\ Correspondence should be addressed to Heng Sun; sunheng@jluat.edu.cn
}

Received 2 November 2021; Revised 4 December 2021; Accepted 18 December 2021; Published 25 January 2022

Academic Editor: Zhihan Lv

Copyright (c) 2022 Heng Sun and Zhe Liu. This is an open access article distributed under the Creative Commons Attribution License, which permits unrestricted use, distribution, and reproduction in any medium, provided the original work is properly cited.

\begin{abstract}
Building Information Modeling (BIM) adoption along with the recent emergence of Internet of Things (IoT) applications provides many unique knowledge and decision-making abilities all through the built environment's life cycle. The ability to connect online sensors utilized in surroundings in real time has led to the definition of the Digital Twin (DT) of the Building Design. The goal of Digital Twins is to synchronize the physical world with a virtual platform for seamless management and control of the construction process, infrastructure solutions, environmental monitoring, and other life span processes within building design. Most of the researchers focused on either BIM or DT in the construction of building application. In this research work, a novel hybrid model of Digital Twin-Building Information Modeling (DT-BIM) is proposed. This model does the process of identifying the shortage of resources, analyzing requirement, performing decision, dispatching the resources, and updating all the process in the database with the support of Artificial Intelligence (AI). Hence, this hybrid model provides improved results when compared to the implementation of the individual technology to the same application. The study results revealed that these hybrid technologies help in assisting the dispatch systems in the construction projects to a greater extent.
\end{abstract}

\section{Introduction}

The construction industry is already one of the largest industries in the world. By 2030, construction output is expected to increase to $\$ 15.5$ trillion, an increase of $85 \%$, with China, the United States, and India leading the way and accounting for $57 \%$ of total growth worldwide, according to the projection [1]. However, the construction industry is still confronted with four challenges: (1) low profitability and productivity; (2) project performance includes budget concerns; (3) a lack of qualified labor; and (4) sustainability issues. Problems like these will persist for some time. The solution is that digital twins can be used as a testing tool [2]. This technology allows for virtual replicating real-world assets, processes, people, and places to be used for a range of different reasons. Before putting anything into production, organizations use Digital Twins to test new assets and procedures as well as to enhance ongoing operations and train people before putting anything into production in the real world, where any problems will be more expensive and more difficult to fix. BIM (Building Information Modeling) is a promising and well-developed theory for managing major healthcare buildings [3]. 3D building elements in BIM give facility managers an easy-to-navigate visual platform with which to retrieve, analyze, and process many types of data. Building Information Modeling-based facility management has had some success. Basic activity logging for routine maintenance was handled using CMMS, which also works well with BIM systems. Although BIM has enabled advancements in the management of building operations, there are still three key challenges, particularly in large medical buildings: Existing software systems do not show 
buildings in real time like they do in the real world. For example, a sudden flood of patients at the hospital's entrance is dangerous and needs to be reported immediately [4]. This platform largely preserves preimported data. No one has access to the most recent status; thus, managers have no way of knowing. It is still difficult to get digital data from various sources. There are numerous differences across systems when it comes to hardware, user interfaces, and data formats to be used. Furthermore, sensor data accumulate rapidly [5]. It is also necessary to improve the methods for transforming and storing such a big amount of data. It is possible to use $3 \mathrm{D}$ models in standard in-use management solutions like BIM platforms to explore or check business data, but this is not possible with 3D models. Offline database analysis would be required to fill this void; however, fast feedback systems would be limited. As a result, rapid decision-making recommendations based on advanced data analysis are essential. To solve these problems, the industrial sector has turned to a relatively new idea known as Digital Twin (DT) [6]. The dispatching system is used for allocating jobs, making calls, coordination, settling payments, and optimizing routes in construction projects. In order to manage these aspects automatically, the study focused on evaluating the performance BIM and Digital Twin and how it helps in intelligent dispatching system in construction projects.

\section{Related Studies}

Building information modeling (BIM) is used for designing the buildings in construction projects. It has been around since the 1970s, just like Digital Twins. It began with research, like so many software projects do. Before it became known as BIM, early researchers such as Chuck Eastman used the term "Building Description System." After a few years of experimenting with Digital Twins, Autodesk and Bentley Systems became well known for their use of the technology in early 2000 [7]. Although BIM has evolved from its earliest days of research, its core objective remains the same. In the early days of BIM, its creators predicted that contractors working on major projects would benefit from having a visual and quantitative representation of their work. They reasoned that ordering supplies and planning projects would be facilitated by it. BIM proves to be as useful as originally thought. AECs (architects, engineers, and contractors) are still being courted by prominent BIM software companies who explain the cost savings of having a primary building reference in a 3D digital model [8]. When working on in-flight projects, this methodology makes it easier to discuss and revise a design. Its advantages include the decrease of project risk due to fewer errors as well as better management of project timelines and budgets. It has a similar sounding name to the Digital Twin, but there are a few important differences between the two.

BIM is used for design and construction collaboration, not for operations and maintenance. BIM is intended to assist architects and builders in the design and construction process, not to create a working model of a building [9]. BIM software, developed to make cooperation easier, can be used to visualize design and construction processes. AECs must understand spatial relationships during the design and prototyping phases of a new construction. Buildings intended for flight rather than ones occupied and used routinely are targeted by this physical information model [10]. A building's design and construction are addressed in BIM. Humans' relationship to their built environment is depicted in a Digital Twin. It is not designed for Real-Time Operational Reaction. Due to the fact that they provide a complete, real-time image of the structure, digital twins are fast becoming the most useful component in a building's technology stack. Data about the current status of construction subsystems, how occupant activity affects those subsystems, and much more can be found with a Digital Twin [11]. With this strategy, the value delivered evolves across the asset's lifetime, increasing over time. In spite of the fact that BIM is a critical data source for any Digital Twin, it cannot answer all of a facility manager's questions about how to run their organization more efficiently. Structures are more important to BIM than humans. A Digital Twin of an Organization or an entire company will be covered by applications in the future, according to Gartner (DTO). It will be possible to add even more context to Digital Twins by incorporating people, processes, and behaviors as data sources. Even during the asset's design and construction, the Digital Twin will surely replace BIM software if trends favor understanding occupants and competing on the quality of the workplace experience [12]. With a focus on people and flexibility, our building information models will need to expand in order for people's behavior patterns and space design to be taken into account. BIM, as previously stated, is unable to produce these outcomes by itself.

By utilizing this technology, oil rigs, production plants, and buildings might all benefit from an increase in safety, efficiency, and regulatory compliance. One of the most important advantages of using a Digital Twin is being able to forecast failures and suggest solutions to avoid them before they happen [13]. Every industry and circumstance in which digital twins are used is growing increasingly relevant. This is due to the increasing prevalence of digital transformation. In other words, cities and structures are becoming smarter as more data are generated and used. By 2021, according to Gartner, there will be IoT endpoints and Digital Twins for potentially billions of scenarios. The builders benefit from enhanced user experience, competitive differentiation, and more. It is becoming increasingly vital for IoT implementation as more IoT platform providers and analytics companies engaged in Digital Twinning technology Design and development from scratch are all part of this process. New building ideas and concepts must be approved by construction designers and developers in order to meet the necessary safety rules, which limit their originality [14]. As a result of time restrictions, software engineers may be limited in the number of concepts they may experiment with. Developers can test their ideas 100 times faster and get them approved 100 times faster using digital simulation that combines all of the crucial real-world characteristics like scale, gravity, and weather. Simulated tests for the safety, feasibility, and long-term viability of new construction 
designs may now be undertaken in the same way as a realworld test and will yield the same results both ways. A datadriven approach to energy management can help save money while also helping the environment because electricity accounts for around 19\% of total construction expenditures. This is made possible thanks to the use of a digital twin [15]. For instance, there are benefits to be had in this situation. More effective use of resources in operations and maintenance of sensor data from the Industrial Internet of Things (IIoT) can be combined with operational and process data to maintain a Digital Twin up to date as assets are deployed or construction projects are finished. Run-time capture records deviations from the optimal process and asset design, and the Digital Twin is instantly updated to reflect these changes.

Predictive learning technology can be used by digital models to foresee asset breakdowns in the future and even suggest ways to prevent them, given the current state of an asset. By anticipating problems before they occur, a Digital Twin is able to prevent them from happening in the real world altogether [16]. Artificial intelligence can be used in the Digital Twin to perform advanced process control, control strategy design, and process optimization. As a result of these tools, a fully digital value loop and integrated lifecycle management can be achieved for engineering asset or plant data. The most cutting-edge tools and procedures combined with internal subject expertise are required to achieve digital transformation in organizations [17]. Thus, new and current data can be contextualized while also delivering useful insights and information to the end user or the reader. Companies may be able to close the loop on process optimization now that they have these new insights. As companies increasingly value information and data, they must begin to embrace digital transformation as an opportunity to transform themselves. To be successful, digital twin initiatives necessitate the establishment of different asset data services, engineering master data, effective visualization tools, and mechanisms for cooperation and workflow for each asset. Assemble a Digital Twin model with reliable information sources and make adjustments at critical control points to improve product or operation performance over the short and long terms. There is a chance predictive maintenance and Digital Twin simulations will help you save money while also reducing the risk of failure [18]. To assist in the development of digital transformation programs and projects, develop a Digital Twin architectural roadmap. It is critical to be able to derive insight from your data. When delivering or launching a project, employ a Digital Twin to evaluate how an organization connects to its existing state and what it will do if something changes internally or externally. Making better and more accurate decisions with the data you have is easier when using a Digital Twin to evaluate process modifications before they are implemented. This provides a plethora of new options. Because of the revolutionary benefits of Digital Twin technology, these companies stand to benefit greatly, as they will be able to maximize their projects' creative potential without adding to their risk profile [19]. Efficiencies in daily management and operational safety become more crucial as the volume and complexity of freshly completed construction projects grow. Elemental problems arise from the combination of static and dynamic aspects, such as the presence of hundreds of rooms, each with a distinct purpose.

According to national rules, China's hospital space is divided into divisions for the general public: medical, clinical, surgical, and also other specialized technology areas. There are numerous factors that must meet strict O\&M quality standards, including temperature, humidity, sewage, exhaust, and air exchange [20]. Complicated crossroads can be created in daily routines by everyone from patients to family members to physicians and office personnel. That is why securing the building appears to be more important than it usually is. Furthermore, many hospital operations in China are delegated to an external team, resulting in additional quality uncertainties and management issues [21]. BIM looks to be more like a single digital shadow and so only partially realizes a digital twin [22]. However, by delivering timely optimization ideas based on the current status mirror, DT can aid decision-making rather than simply reflecting it. Services such as energy forecasting, failure prediction, and operation guidance are all improved by the building's lifetime service through the use of real-time monitoring (RTM) [23]. This study evaluates how DT and BIM help in developing intelligent dispatch system in the construction projects.

2.1. Drawbacks of the Study. The field of Digital Twins for such physical Environment is still very much in infant stages, as well as the need to recognize the developments in the fundamental technological solutions and maintain a convergent frame of reference for long-term development research. This document performed a comprehensive review to recognize the advancement of technological innovations that aid in the transformation of BIM to Digital Twins in building design applications. Nevertheless, investigation in Digital Twins for the Physical Environment is still in its infancy, as well as the need to recognize advances within fundamental technological solutions as well as to establish a concurrent context for future expansion investigation.

2.2. Objectives of the Study. A novel Digital Twin-Building Information Modeling (DT-BIM) hybrid model is proposed. With the help of Artificial Intelligence, this model identifies resource shortages, analyzes requirements, makes decisions, dispatches resources, and updates all processes in the database (AI). As a result, when compared to the implementation of the individual technology to the same application, this hybrid model produces better results. The study's findings revealed that these hybrid technologies aid dispatched systems in construction projects to a greater extent.

\section{Methods}

Digital Twin (DT) is a technology that aids in the easy access of any user-defined application with ease. This trending technology is a combination of software and hardware 
implementation for a given on-demand application; and this is a specialized property of the technology. Furthermore, DT works with the support of various sensors required for identification of the required material along with the Internet of Things (IoT) [24]. Hence, it will be a best future technology. On the other hand, another trending technology is Building Information Modeling (BIM). The unique property of this BIM technology is to provide a visual representation of the building with an advanced illustration of physical and functional characteristics of the given place or the building [25]. These two technologies with their advanced features will make the building construction an easier task. Henceforth, in this research work, a novel hybrid model of these two technologies named Digital Twin-Building Information Modeling (DT-BIM) is proposed and the results were discussed.

Figure 1 represents the proposed architectural model of the hybrid DT-BIM model. In the proposed model, BIM supports the prediction support for the simulation work and the AI-based visualization along with the data storage. This AI-based visualization works with the support of DT technology. The predicted or the simulated results are stored in the database for the future prediction process. In the DTBIM model, two major blocks are used to simulate the dispatching of the required construction materials, and sensors to identify the requirements. In the first block, the decision on the Construction Materials Dispatch (CMD) is performed with the analysis of energy consumption, cost, and the comfortability of the end user who will be occupying the building. After making the analysis with the support of BIM, the materials will be dispatched to the construction site and all the details will be updated in the database. In the Real Building (RB) block, all the required sensors will be fixed in the construction area to analyze the shortage of the construction materials and also certain sensors to identify the issues in the building (if any). These details will be updated in the database along with the data transfer to the CMD block for further processes.

3.1. Digital Twin-BIM-Hybrid Model. To recognize emerging technologies that will aid in the transformation of BIM to Digital Twins in this as the authors created a level ladder classification structure based also on building life cycle to match the actual state-of-the-art in Digital Twin applications in order to systematically classify the multiple studies, implementations were further classified in each level of this taxonomy depending on the study areas.

The difficult part is determining what a delegate test implies and how small or large it must be.

$$
\begin{aligned}
S_{m l} & =\operatorname{dig}_{s 2 I}^{\max } T\left(I_{Q} ; T_{R}: S, t^{*}\right), \\
S_{\text {map }} & =\operatorname{dig}_{s 2 I}^{\max } P\left(S \vdots I_{Q} ; T ; t^{*}\right),
\end{aligned}
$$

where $I_{Q}$ and $T_{R}$ are the sets of afflicted and shielded access points at the time and the constants $\mathrm{P}$ and $\mathrm{Q}$ represent the number of infectious and shielded access points up to time $t$.

It omits time ' $s$ ' for matrix multiplication clarity.

To ascertain the generator $S$,

$$
\begin{aligned}
S_{\text {map }} & =\operatorname{dig}_{s 2 I}^{\max } T\left(S \vdots I_{Q} ; T_{R} ; t^{*}\right) \\
& =\operatorname{dig}_{s 2 I}^{\max } \frac{T\left(I_{Q} ; T_{R} \vdots S, p^{*}\right) T\left(S, t^{*}\right)}{T\left(I_{Q} ; T_{R} ; t^{*}\right)} \\
& =\operatorname{dig}_{s 2 I}^{\max } T\left(I_{Q} ; T_{R} \vdots S, t\right) \cdot T\left(S, t^{*}\right),
\end{aligned}
$$

where $S: I_{Q}, T_{R}$, and $t^{*}$ represent the maximum number of possible propagations for the same probability occurring in the information source and $T\left(S t^{*}\right)$ represents the probability and classifies for the information source.

In the BIM model, $T\left(I_{Q} ; T_{R} \vdots S t\right)$ is the probability which has the realisations $I_{Q}$ and $T_{R}$ occurring in a source of information $v$ and $p^{*}$ denotes protective. As a result, if $T\left(S t^{*}\right)$ is assumed to be homogeneous over, BIM is equivalent to DT (Digital Twin) and BIM is used to detect the probability:

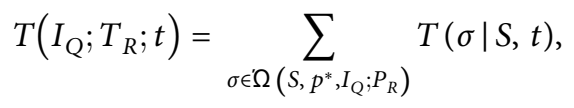

where $\Omega\left(v, t^{*}, I_{Q}, T_{R}\right)$ is the set of all likely dissemination classifications given $I_{Q}$ and $T_{R}$.

Let $\mathrm{G}$ be consistent.

Based on the $K$ representation for the number of possible propagation sequences, the same $\sigma$ approach as a source of the information in DT and BIM is used:

$$
\begin{aligned}
S_{m l} & =\operatorname{dig}_{s \in I_{Q}}^{\max } K\left(S, t, I_{Q}, T_{R}\right), \\
S_{\text {map }} & =\operatorname{dig}_{s \in I_{Q}}^{\max } K\left(S, t, I_{Q}, T_{R}\right) \cdot P\left(S, t^{*}\right),
\end{aligned}
$$

where

$K\left(S, t, I_{Q}, T_{R}\right)=\left|\Omega\left(S, t^{*}, I_{Q}, T_{R}\right)\right|=O(R+Q) ! \prod_{\mu \varepsilon I_{Q} \cup P_{R}}\left|T_{\mu}^{v}\right|-1$.

This presumption for the $\Omega$ variety of feasible propagation sequential nodes provides both information and context around the same time for $S_{m l}$ as well as $S_{\text {map }}$.

It calls for $S_{m l}$ and $S_{\text {map }}$ in the propagation center. Let $\left|T_{\mu}^{v}\right|$ be the number of requirements. The subtree $T_{\mu}^{v}$ rooted at requirements. Let $v$ be the material foundation and BIM and digital twin communication take place between $i$ and $j$.

Let $v$ be the material foundation and BIM and digital twin communication take place between $i$ and $j$. Place it at the heart of the framework. Let $S(t)$ denote the network's sensor set:

$$
S(t)=S(t-1)+\beta^{*} a(t) .
$$

There at connection, the $a(t)$ transverse and longitudinal roads may become fractious. The network elements are expected to move directly ahead, $S(t-1)$ turn left as well as right, with a guaranteed connection, where

$$
-1 \leq \beta \leq 1 .
$$

If it is below zero, it indicates that the node is an impacting counter (negative acceleration). Otherwise, it moves at a faster pace. 


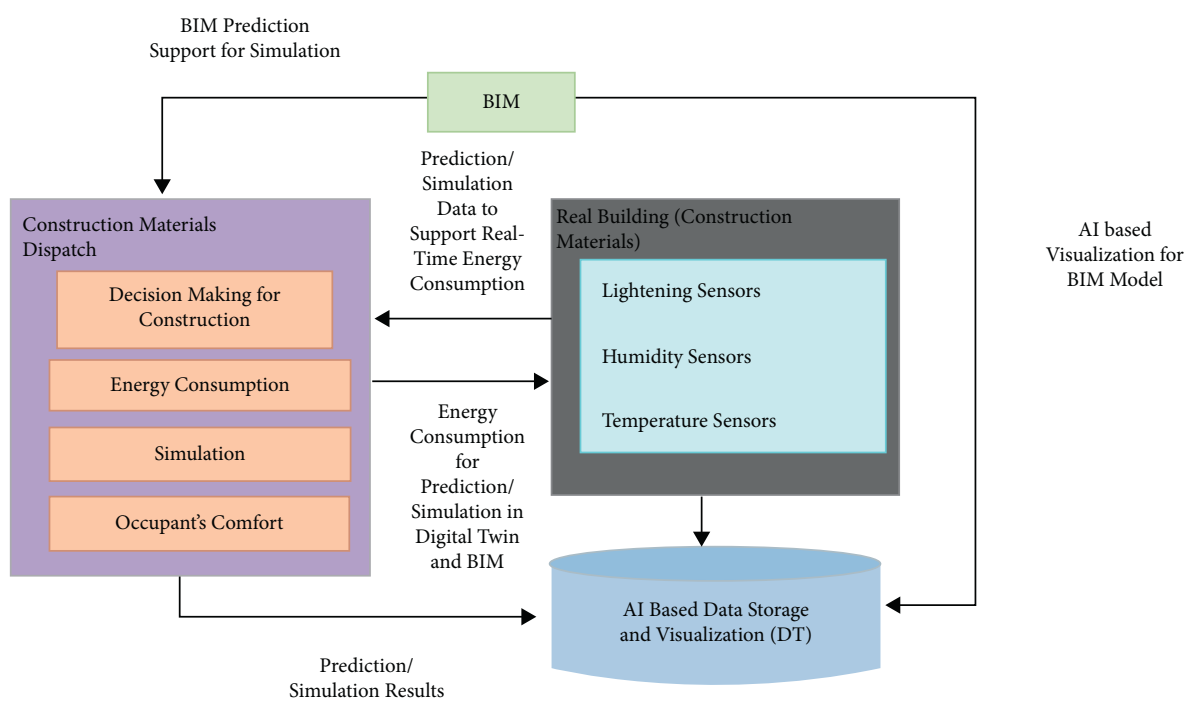

Figure 1: Proposed architecture.

At time $t$, the remaining battery energy $(R 1)$ and the ingested energy $(\mathrm{C} 1)$ of an access point are being shown elsewhere here:

$$
\begin{aligned}
\beta_{i(t)} & =\frac{R 1_{i(t)}}{C 1_{i(t)}}, \\
B I M & =\frac{\left(T^{i} n d\right) \beta}{\sum j \in Q^{i} d\left(T^{i} j d\right) \beta}, \quad \beta \geq 1, \\
\text { if } \mathrm{s}(\mathrm{t}) & <\operatorname{Smax} \text { Then } \mathrm{s}(\mathrm{t})=\operatorname{Smax}, \\
\text { if } \mathrm{s}(\mathrm{t}) & <\operatorname{Smin} \text { Then } \mathrm{s}(\mathrm{t})=\operatorname{Smin}, \\
C 1_{R 1} & =\sum_{j=1}^{i=1} C 1(i) .
\end{aligned}
$$

\section{Results and Discussion}

In general, scan-to-BIM refers to the process of converting 3D scanning point cloud information from data building structures into a BIM model. Academically, the application of bitmap image technology in construction is still in its early stages, with no unified standard or implementation mode in place. The scan-to-BIM scanner parameters for accuracy $(15 \mathrm{~mm})$, positional accuracy $(15 \mathrm{~mm})$, and coverage $(85$ percent) are used to evaluate data collection quality (refer Figure 2). Coverage is determined once the scanned building meets the scanning accuracy and also resolution satellite requirements (Table 1).

It is significant to assess the AI technology retrofitting scheme for existing buildings using a digital twin, which can improve the energy efficiency as well as reduce carbon emissions from buildings. This paper examines in depth the effect of photovoltaic solar module configuration angle on energy consumption in buildings and photovoltaic device electricity production. From Figure 3, the performance analysis of energy consumption using different models has been represented (Table 2).

The scan-to-BIM-based digital twin assessment AI method is suggested in analyses and simulates the impact of the environment building refitting schemes on energy consumption in buildings and photovoltaic electricity production, and the resulting changes contain similar energy consumption. From Figure 4, we can see descriptive statistics of digital twin and BIM (Table 3).

The real-time energy monitoring and management of such a building are essential for creating effective and sustainable structures. Building energy quality management can benefit from BIM and DT applications. Creating a realtime visualization of energy consumption in buildings that resulted in a $17 \%$ energy savings attributable to easier data acquisition resulted in improved control of lighting control systems (refer Figure 5), likewise, by integrating BIM and DT gadgets via open sending messages specifications and AI technology (Table 4).

The viability of using AI techniques to predict the infrastructure based on data from the current model was discussed, as were the methodologies as well as challenges of smart building Digital Twins, that also suggested to integrate BIM to analyze the energy usage of buildings. From Figure 6, we can observe that the performance of digital twins performs well compared with that of BIM and DT. It indicates that our hybrid model is suggested to improve the dispatching process easier (Table 5).

It can be seen that a substantial percentage of the previous studies were classified as meaning that many of the previous application areas of "Digital Twins," beginning with BIM-supported simulations for tasks such as 4D building lifecycle simulations and enhancing energy evaluation, have been shown to help with evaluation of the building's lifecycle and accomplish a much more efficient and environmentally building. 


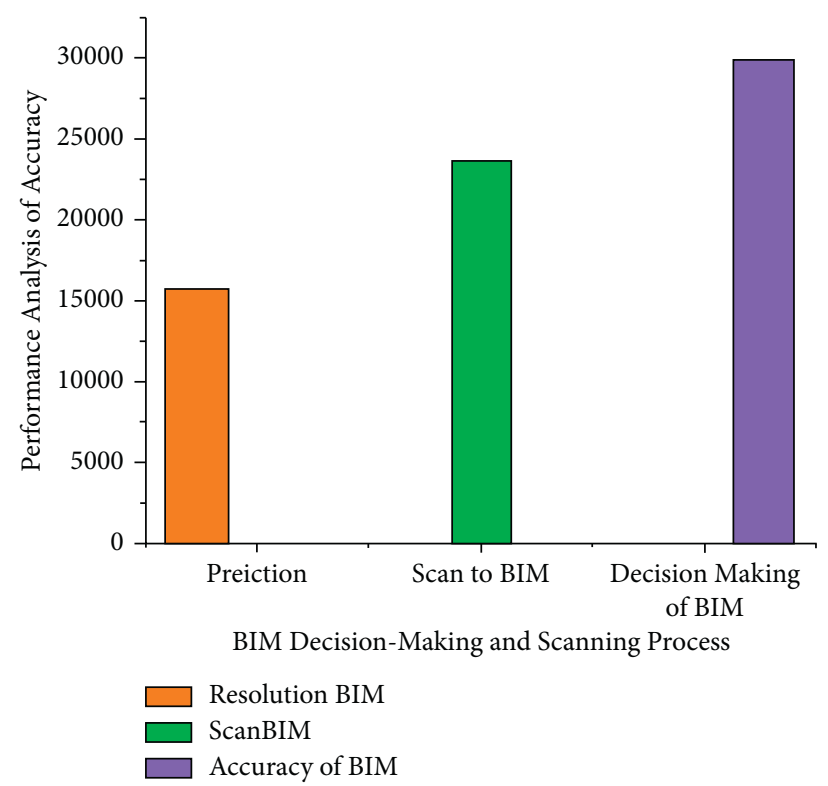

FiguRE 2: BIM scanning process.

TABLE 1: BIM scanning process.

\begin{tabular}{lcccc}
\hline & \multicolumn{3}{c}{ BIM decision-making and scanning process } \\
Number & Object & Accuracy $(\mathrm{mm})$ & Resolution $(\mathrm{mm})$ & Coverage $(\%)$ \\
\hline 1 & East & 1.5 & $0.6-15$ & 100 \\
2 & West & 1.5 & $0.6-7$ & 100 \\
3 & North & 1.5 & $0.5-6$ & 100 \\
4 & South & 1.5 & $0.6-4$ & 100 \\
\hline
\end{tabular}

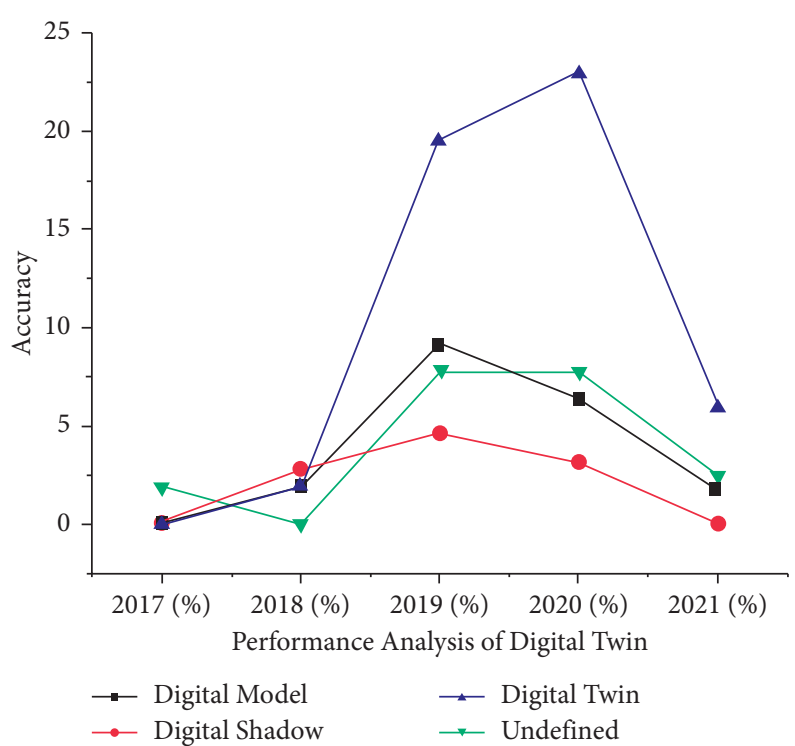

Figure 3: Performance analysis for digital twin with artificial intelligence.

TABLE 2: Performance result analysis for digital twin with AI.

\begin{tabular}{|c|c|c|c|c|c|c|}
\hline \multirow{2}{*}{ Data type } & \multicolumn{5}{|c|}{ Year } & \multirow{2}{*}{ Total (\%) } \\
\hline & $2017(\%)$ & $2018(\%)$ & $2019(\%)$ & $2020(\%)$ & $2021(\%)$ & \\
\hline Digital model & 0.01 & 1.92 & 9.23 & 6.34 & 1.78 & 19.28 \\
\hline Digital shadow & 0.01 & 2.76 & 4.67 & 3.12 & 0.01 & 10.57 \\
\hline Digital twin & 0.01 & 1.93 & 19.45 & 22.92 & 5.92 & 50.23 \\
\hline Undefined & 1.89 & 0.01 & 7.78 & 7.78 & 2.45 & 19.91 \\
\hline Total & 1.92 & 6.62 & 41.13 & 40.14 & 10.16 & 100 \\
\hline
\end{tabular}




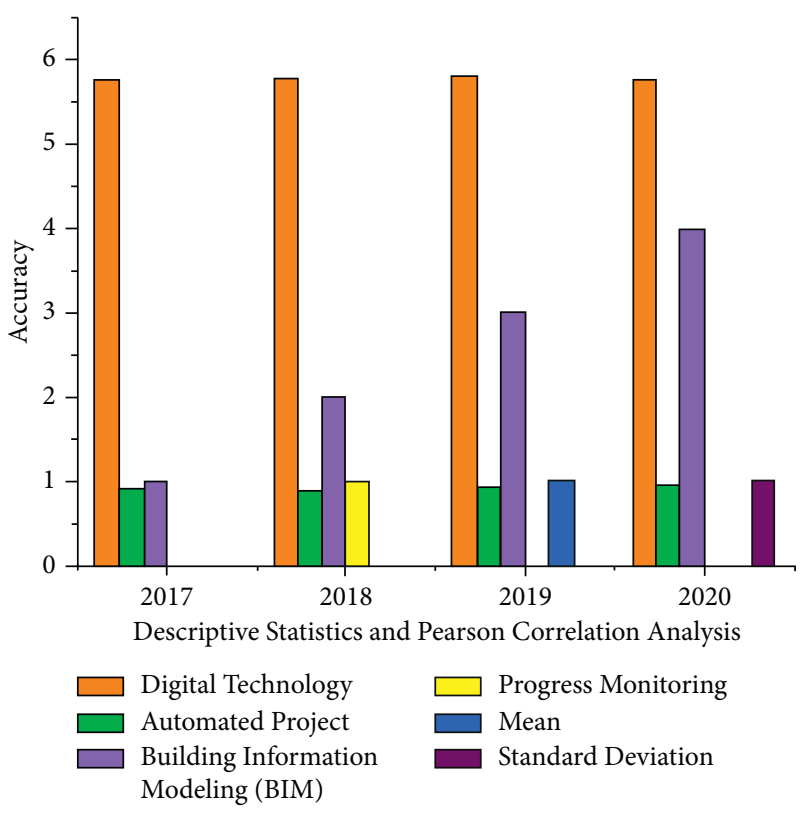

FIgUre 4: Descriptive statistics and Pearson correlation analysis of digital twin and BIM.

TAвLe 3: Descriptive statistics and Pearson correlation analysis.

\begin{tabular}{lccccc}
\hline Parameters & Mean & Standard deviation & 1 & 2 & 3 \\
\hline Digital technology & 5.76 & 0.92 & 1.00 & & \\
Building information modeling (BIM) & 5.75 & 0.91 & $0.7564^{*}$ & 1.00 & \\
Automated project & 5.79 & 0.93 & $0.7634^{*}$ & $0.812^{* *}$ & 1.00 \\
Progress monitoring & 5.74 & 0.96 & $0.834^{* *}$ & $0.845^{* *}$ & $0.837^{* *}$ \\
\hline
\end{tabular}

Note: ${ }^{*} p<0.1,{ }^{* *} p<0.05,{ }^{* * *} p<0.01$, and $n=346$.

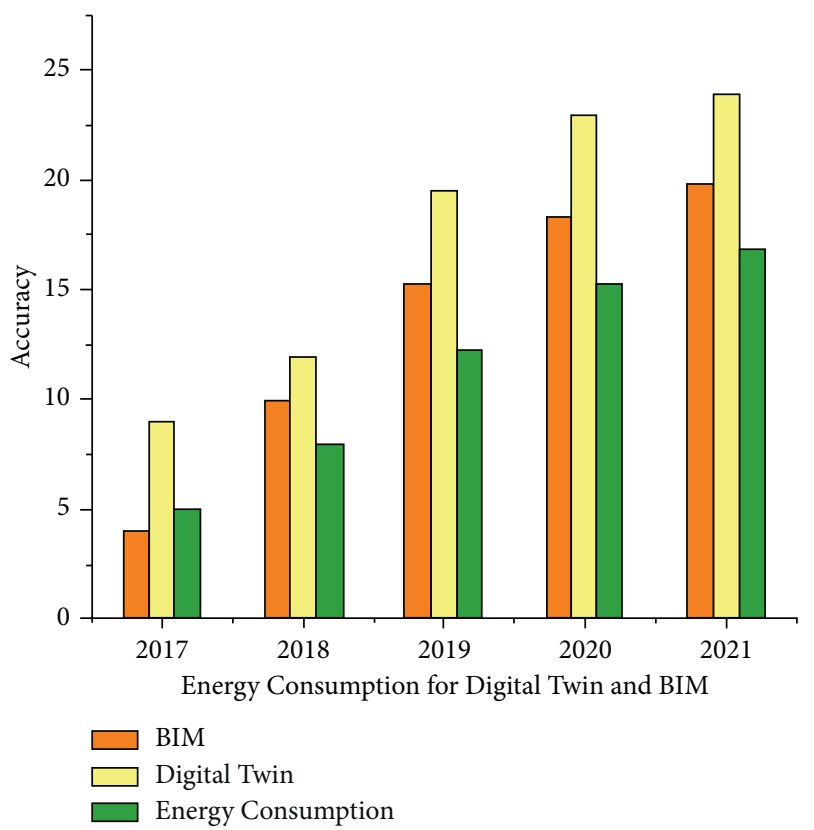

Figure 5: Energy consumption for Digital Twin and BIM using AI technology.

4.1. Future Direction of the Proposed Research. The different algorithms used in BIM technology in construction to improve the visualization of the building project mostly to avoid building collisions at an earlier stage. In the building construction industry, Digital Twins play an important role in gathering information about the organization using 
TABLE 4: Result analysis for energy consumption for digital twin and BIM using AI technology.

\begin{tabular}{lccc}
\hline Years & Energy consumption for digital twin and BIM & Digital twin & $\begin{array}{c}\text { Energy } \\
\text { consumption }\end{array}$ \\
2017 & BIM & 09.78 & 15.89 \\
2018 & 10.34 & 14.56 & 7.92 \\
2019 & 16.76 & 19.12 & 9.23 \\
2020 & 17.36 & 25.87 & 14.67 \\
2021 & 19.67 & 26.32 & 16.12 \\
\hline
\end{tabular}

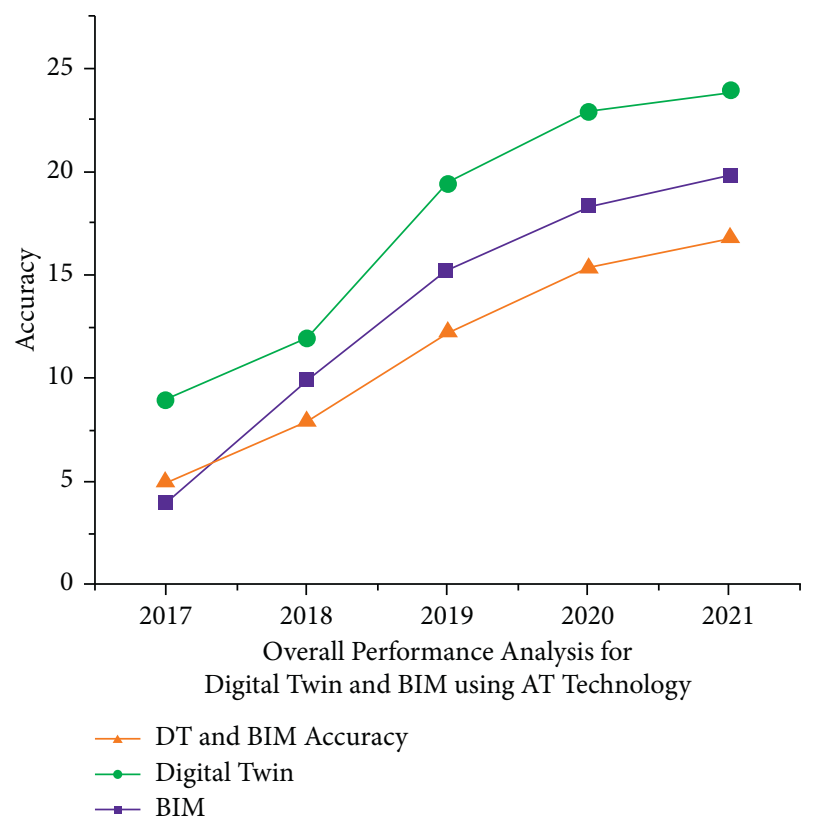

FIGURE 6: Overall performance analysis for Digital Twin and BIM using AI technology.

TABLE 5: Overall performance result analysis for Digital Twin and BIM using AI technology.

\begin{tabular}{lccc}
\hline Years & \multicolumn{3}{c}{$\begin{array}{c}\text { Performance analysis for digital twin and BIM } \\
\text { using AI technology } \\
\text { Digital twin }\end{array}$} \\
\hline 2017 & BIM & 9.01 & DT with BIM \\
2018 & 4.01 & 11.93 & 5.14 \\
2019 & 9.92 & 19.45 & 7.92 \\
2020 & 15.23 & 22.92 & 12.23 \\
2021 & 18.34 & 23.92 & 15.34 \\
\hline
\end{tabular}

advanced technology and the Internet of Things (IoT). So, the intelligence obtained is used to supervise the resources in the constructed building, schedule maintenance, and much more. BIM facilitates a material selection by utilizing previously collected data.

\section{Conclusions}

Digital Twin and BIM are new emerging technologies that are implemented in all the industrial sectors. In this research work, these technologies are implemented for the construction sector. Digital Twin is a technology that includes the implementation of the application from the software to the hardware. On the other hand, BIM is a technology in the construction sector providing the visualization of the building project to avoid collision of the buildings at the earlier stage. In the building construction sector, Digital Twin plays a significant role in collecting information about the building with the Internet of Things (IoT). The utilization of the information gathered aids in monitoring the assets in the constructed building, scheduling maintenance, and much more. BIM supports the selection of materials with the help of earlier collected information. In this research work, a novel hybrid algorithm named DT-BIM is proposed for combining the two technologies for the better construction and maintenance of the buildings. From the results of the hybrid technology of DT-BIM, this hybrid model provides better results in the dispatch of the required construction materials to the building construction location than the individual technology. It can be concluded that the future of the building construction will depend on these two technologies. As a result, when compared to such a successful execution of an individual technology to the very same application, such a hybrid model provides more accurate results. The results of this analysis of accuracy in (97\%) revealed that all these hybrid technologies aid delivery systems in building projects to a larger extent.

\section{Data Availability}

The data used to support the findings of this study are available from the corresponding author upon request.

\section{Conflicts of Interest}

The authors declare that there are no conflicts of interest.

\section{References}

[1] A. Watson, "BIM leaders of the future: engaging the digital generation," Construction Manager's BIM Handbook, John Wiley \& Sons, Hoboken, NJ, USA, pp. 133-141, 2016.

[2] J. Eynon, "Afterword: BIM, digital life and the third industrial revolution," Construction Manager's BIM Handbook, John Wiley \& Sons, Hoboken, NJ, USA, pp. 146-150, 2016.

[3] L.-S. Kang, H.-S. Moon, H.-S. Kim, and J.-M. Kwak, "Usability improvement of BIM for construction projects using active BIM functions," Korean Journal of Construction Engineering and Management, vol. 14, no. 5, pp. 74-83, 2013.

[4] P. Barnes, "The BIM Tender Stage," in BIM for Project Managers, pp. 39-55, ICE Publishing, London, UK, 2020. 
[5] P. Barnes, "The BIM Management Roles," in BIM for Project Managers, pp. 33-38, ICE Publishing, London, UK, 2020.

[6] M. Deng, C. C. Menassa, and V. R. Kamat, "From BIM to digital twins: a systematic review of the evolution of intelligent building representations in the AEC-FM industry," Journal of Information Technology in Construction, vol. 26, pp. 58-83, 2021.

[7] P. Barnes, "BIM Monitoring and Control," in BIM for Project Managers, pp. 119-126, ICE Publishing, London, UK, 2020.

[8] G. Kapogiannis and A. Mlilo, "Digital Construction Strategies and BIM in Railway Tunnelling Engineering," in Tunnel Engineering - Selected Topics, IntechOpen, London, UK, 2020.

[9] D. Lee, S. H. Lee, N. Masoud, M. S. Krishnan, and V. C. Li, "Integrated digital twin and blockchain framework to support accountable information sharing in construction projects," Automation in Construction, vol. 127, Article ID 103688, 2021.

[10] F. Tao, M. Zhang, and A. Y. C. Nee, "Digital twin and big data," Digital Twin Driven Smart Manufacturing, Elsevier, Amsterdam, Netherlands, pp. 183-202, 2019.

[11] R. Sacks, I. Brilakis, E. Pikas, H. S. Xie, and M. Girolami, "Construction with Digital Twin Information Systems," DataCentric Engineering, vol. 1, 2020.

[12] Y. Wang, A. Liu, F. Tao, and A. Y. C. Nee, "Digital twin driven conceptual design," Digital Twin Driven Smart Design, Elsevier, Amsterdam, Netherlands, pp. 33-66, 2020.

[13] L. Wang, F. Tao, A. Liu, and A. Y. C. Nee, "Digital twin driven design evaluation," Digital Twin Driven Smart Design, Elsevier, Amsterdam, Netherlands, pp. 139-164, 2020.

[14] V. Jain, N. Luthra, and D. Saini, "Digital twin technology," Digital Twin Technology, CRC Press, Boca Raton, FL, USA, pp. 1-21, 2021.

[15] A. Costin, A. Adibfar, H. Hu, and S. S. Chen, "Building Information Modeling (BIM) for transportation infrastructure - literature review, applications, challenges, and recommendations," Automation in Construction, vol. 94, pp. 257-281, 2018.

[16] L. Barazzetti, M. Gianinetto, and M. Scaioni, "Automatic processing of many images for 2D/3D modelling," Digital Transformation of the Design, Construction and Management Processes of the Built Environment, Springer International Publishing, Berlin, Germany, pp. 355-365, 2019.

[17] M. Zhang, F. Sui, A. Liu, F. Tao, and A. Y. C. Nee, "Digital twin driven smart product design framework," Digital Twin Driven Smart Design, Elsevier, Amsterdam, Netherlands, pp. 3-32, 2020.

[18] S. Fabozzi, S. A. Biancardo, R. Veropalumbo, and E. Bilotta, "I-BIM based approach for geotechnical and numerical modelling of a conventional tunnel excavation," Tunnelling and Underground Space Technology, vol. 108, Article ID 103723, 2021.

[19] C. I. Papanagnou, "A Digital Twin Model for Enhancing Performance Measurement in Assembly Lines," in Internet Of Things, pp. 53-66, Springer International Publishing, Berlin/ Heidelberg, Germany, 2019.

[20] S. A. Biancardo, A. Capano, S. G. de Oliveira, and A. Tibaut, "Integration of BIM and procedural modeling tools for road design," Infrastructure, vol. 5, no. 4, p. 37, 2020.

[21] A. Grosso, "Reliability of SHM procedures and decision support in infrastructure management," Bridge Maintenance, Safety, Management and Life-Cycle Optimization, p. 322, CRC Press, Boca Raton, FL, USA, 2010.

[22] M.-H. Shin and J.-H. Baek, "Design and construction of rail infrastructure BIM integrated management system for systematic management of rail infrastructure BIM design performance products," Journal of the Korean Society for Railway, vol. 23, no. 9, pp. 886-894, 2020.

[23] C. Boddupalli, A. Sadhu, E. Rezazadeh Azar, and S. Pattyson, "Improved visualization of infrastructure monitoring data using building information modeling," Structure and Infrastructure Engineering, vol. 15, no. 9, pp. 1247-1263, 2019.

[24] M. U. Khalid, M. K. Bashir, and D. Newport, "Development of a building information modelling (BIM)-Based real-time data integration system using a building management system (BMS)," Building Information Modelling, Building Performance, Design and Smart Construction, Springer International Publishing, Berlin, Germany, pp. 93-104, 2017.

[25] K. K. Han and M. Golparvar-Fard, "Appearance-based material classification for monitoring of operation-level construction progress using 4D BIM and site photologs," Automation in Construction, vol. 53, pp. 44-57, 2015. 